q-alg/9605011

\title{
General methods for constructing bispectral operators
}

\author{
B. Bakalov * E. Horozov ${ }^{\dagger}$ M. Yakimov ${ }^{\ddagger}$ \\ Department of Mathematics and Informatics, \\ Sofia University, 5 J. Bourchier Blvd., Sofia 1126, Bulgaria
}

\begin{abstract}
We present methods for obtaining new solutions to the bispectral problem. We achieve this by giving its abstract algebraic version suitable for generalizations. All methods are illustrated by new classes of bispectral operators.
\end{abstract}

\section{Introduction}

The bispectral problem of J. J. Duistermaat and F. A. Grünbaum [9] consists of finding all bispectral ordinary differential operators, i.e. operators $L\left(x, \partial_{x}\right)$ having a family of eigenfunctions $\psi(x, z)$, which are also eigenfunctions for another differential operator $\Lambda\left(z, \partial_{z}\right)$ in the spectral parameter:

$$
\begin{aligned}
& L\left(x, \partial_{x}\right) \psi(x, z)=f(z) \psi(x, z), \\
& \Lambda\left(z, \partial_{z}\right) \psi(x, z)=\theta(x) \psi(x, z) .
\end{aligned}
$$

In [3] we constructed large families of solutions to this problem, generalizing all previously known results (cf. [9, 19, 16, 17, 13], etc.). We obtained them as special Darboux transformations (called "polynomial") from the most obvious solutions the (generalized) Bessel and Airy ones. We recall that the Bessel (respectively Airy) operators have the form $L=x^{-N} P\left(x \partial_{x}\right)$ (respectively $L=P\left(\partial_{x}\right)-x$ ) for some polynomial $P$ of degree $N$.

For fixed $\psi$, all operators $L\left(x, \partial_{x}\right)$ for which $\psi$ is an eigenfunction form a commutative algebra $\mathcal{A}_{\psi}$, called the spectral algebra. Then $\operatorname{Spec} \mathcal{A}_{\psi}$ is an algebraic curve [6] - the spectral curve. The dimension of the space of eigenfunctions $\psi$ (= g.c.d. $\operatorname{ord} L, L \in \mathcal{A}_{\psi}$ ) is called a rank of $\mathcal{A}_{\psi}$. Following G. Wilson [17] we call the spectral algebra $\mathcal{A}_{\psi}$ bispectral iff there exists an operator $\Lambda\left(z, \partial_{z}\right)$ satisfying (0.2).

The purpose of this paper is to present general methods for constructing bispectral operators (respectively - algebras). Some of our statements are only abstract

\footnotetext{
*E-mail: bbakalov@fmi.uni-sofia.bg

${ }^{\dagger}$ E-mail: horozov@fmi.uni-sofia.bg

${ }^{\ddagger}$ E-mail: myakimov@fmi.uni-sofia.bg
} 
versions of concrete results [9, 17, 3, 14]. But adopting the algebraic point of view enables us to clarify the ideas and to give many classes of new examples of bispectral operators. Moreover, being purely algebraic, some of the methods work also for difference or $q$-difference analogs. The latter case is illustrated here by many examples but more complete theory in the spirit of [3] will be considered elsewhere.

In the process of our work [3] we substantially used the bispectral involution $b$ introduced by G. Wilson [17. It is, roughly speaking, the map exchanging the roles of the arguments in the wave function $\psi(x, z)$ :

$$
b \psi(x, z)=\psi(z, x) .
$$

The main idea which we exploit here is to consider the bispectral involution as an anti-isomorphism of algebras of ordinary differential operators (cf. [14]), or more generally - arbitrary rings.

Our first construction of new bispectral algebras from old ones is based on exhibiting anti-isomorphisms of algebras as compositions of already known ones and inner automorphisms. In particular, we find new bispectral algebras of arbitrary non-prime rank with both spectral curves (in $x$ and in $z$ ) isomorphic to $\mathbb{C}$.

The second construction is an abstract version - which again uses only the bispectral anti-isomorphism - of the bispectrality theorem from [3]. Analizing the latter has led us to the notion of bispectral Darboux transformations which is applicable to other (than differential) operators.

At the end we consider sequences of Darboux transformations depending on integers parametrizing the elements of the sequence. Then allowing the parameters to be any complex numbers we again get new solutions to the bispectral problem.

\section{Bispectral triples}

Here we present a general formulation of the bispectral problem which works not only for differential but also for difference or $q$-difference modifications of the original problem of [9].

Let $\mathcal{R}$ and $\mathcal{R}^{\prime}$ be associative algebras over a field $k$ and let $\mathcal{M}$ be a left module over both of them. Let $\mathcal{K}$ and $\mathcal{K}^{\prime}$ be fields such that $k \subset \mathcal{K} \subset \mathcal{R}$ and $k \subset \mathcal{K}^{\prime} \subset \mathcal{R}^{\prime}$.

Definition 1.1 We call an element $L \in \mathcal{R}$ bispectral iff there exist $\psi \in \mathcal{M}, \Lambda \in \mathcal{R}^{\prime}$, $f \in \mathcal{K}^{\prime}, \theta \in \mathcal{K}$ such that

$$
\begin{array}{r}
L \psi=f \psi, \\
\Lambda \psi=\theta \psi .
\end{array}
$$

In order to have non-trivial problem we assume that

$$
L \psi=0 \text { implies } L=0 \text { for } L \in \mathcal{R}\left(\text { or } \mathcal{R}^{\prime}\right) \text {. }
$$

Let us fix $\psi \in \mathcal{M}$ satisfying (1.1) and (1.2). We are interested in the equation (cf. [14])

$$
P \psi=Q \psi
$$


for $P \in \mathcal{R}, Q \in \mathcal{R}^{\prime}$. We put

$$
\begin{aligned}
& \mathcal{B}_{\psi}=\left\{P \in \mathcal{R} \mid \exists Q \in \mathcal{R}^{\prime} \text { for which (1.4) is satisfied }\right\} \\
& \mathcal{B}_{\psi}^{\prime}=\left\{Q \in \mathcal{R}^{\prime} \mid \exists P \in \mathcal{R} \text { for which (1.4) is satisfied }\right\} .
\end{aligned}
$$

We shall assume that the actions of $\mathcal{R}$ and $\mathcal{R}^{\prime}$ on $\mathcal{M}$ commute and that (1.3) holds. Then $\mathcal{B}_{\psi}$ and $\mathcal{B}_{\psi}^{\prime}$ are associative algebras over $k$ without zero divisors. Obviously (1.4) defines an anti-isomorphism

$$
b: \mathcal{B}_{\psi} \rightarrow \mathcal{B}_{\psi}^{\prime}, \quad b(P)=Q .
$$

Introduce also the subalgebras

$$
\begin{gathered}
\mathcal{K}_{\psi}=\mathcal{B}_{\psi} \cap \mathcal{K}, \quad \mathcal{K}_{\psi}^{\prime}=\mathcal{B}_{\psi}^{\prime} \cap \mathcal{K}^{\prime}, \\
\mathcal{A}_{\psi}=b^{-1}\left(\mathcal{K}_{\psi}^{\prime}\right), \quad \mathcal{A}_{\psi}^{\prime}=b\left(\mathcal{K}_{\psi}\right) .
\end{gathered}
$$

Then $\mathcal{A}_{\psi}$ is a commutative algebra isomorphic to $\mathcal{K}_{\psi}^{\prime}$ (the spectral algebra). With a tilde we shall denote the fields of quotients of these commutative rings, e.g. $\mathcal{K}_{\psi} \subset$ $\widetilde{\mathcal{K}}_{\psi} \subset \mathcal{K}$. Then $b$ extends to isomorphisms $\widetilde{\mathcal{K}}_{\psi} \rightarrow \widetilde{\mathcal{A}}_{\psi}^{\prime}$ and $\widetilde{\mathcal{A}}_{\psi} \rightarrow \widetilde{\mathcal{K}}_{\psi}^{\prime}$.

Definition 1.2 We call the triple $\left(\mathcal{B}_{\psi}, \mathcal{B}_{\psi}^{\prime}, b\right)$ a bispectral triple iff both $\mathcal{K}_{\psi}$ and $\mathcal{K}_{\psi}^{\prime}$ contain non-zero elements.

Obviously if $\left(\mathcal{B}_{\psi}, \mathcal{B}_{\psi}^{\prime}, b\right)$ is a bispectral triple then any element $L \in \mathcal{A}_{\psi}$ is bispectral.

Example 1.3 (i) Let $\mathcal{R} \cong \mathcal{R}^{\prime}$ be two copies of the rings of differential operators of one variable with rational coefficients and let $\mathcal{M}$ be a module of differentiable functions $\psi(x, z)$ (or formal power series) on which $\mathcal{R}$ acts on the variable $x$ and $\mathcal{R}^{\prime}$ acts on the variable $z$. Put $\psi(x, z)=\delta(x-z)$ (Dirac delta function). Then $\mathcal{B}_{\psi}$ and $\mathcal{B}_{\psi}^{\prime}$ are two copies of the Weyl algebra of the differential operators with polynomial coefficients and $b$ is the formal conjugation $*$, i.e. $b(x)=z, b\left(\partial_{x}\right)=-\partial_{z}$.

(ii) [3, 14] Let $L_{\alpha}$ be the (generalized higher) Airy operator

$$
L_{\alpha}=\partial^{N}+\sum_{i=2}^{N-1} \alpha_{i} \partial^{N-i}-x, \quad \alpha \in \mathbb{C}^{N-2}
$$

and let $\Phi(x)$ be a solution of the equation $L_{\alpha} \Phi=0$. Put $\psi(x, z)=\Phi(x+z)$. Then $\mathcal{B}_{\psi} \cong \mathcal{B}_{\psi}^{\prime}$ are again two copies of the Weyl algebra but now $b$ is defined via

$$
b(x)=L_{\alpha}, \quad b(\partial)=\partial .
$$

Then $\mathcal{K}_{\psi}=\mathbb{C}[x], \quad \mathcal{A}_{\psi}=\mathbb{C}\left[L_{\alpha}\right]$.

(iii) [3] Let $L_{\beta}$ be the (generalized) Bessel operator

$$
L_{\beta}=x^{-N}\left(D-\beta_{1}\right) \cdots\left(D-\beta_{N}\right), \quad \beta \in \mathbb{C}^{N},
$$

where $D=x \partial$. Let $\Phi(x)$ be a solution of the equation $L_{\beta} \Phi(x)=\Phi(x)$ and $\psi(x, z)=$ $\Phi(x z)$. Then for "generic" $\beta$ (see [3]) $\mathcal{B}_{\psi} \cong \mathcal{B}_{\psi}^{\prime}$ is generated by $x^{N}, D, L_{\beta}$; now $b\left(x^{N}\right)=L_{\beta}, b(D)=D, b\left(L_{\beta}\right)=x^{N}$ and $\mathcal{K}_{\psi}=\mathbb{C}\left[x^{N}\right], \mathcal{A}_{\psi}=\mathbb{C}\left[L_{\beta}\right]$. 
Example 1.4 (i) Let $\mathcal{R}$ and $\mathcal{R}^{\prime}$ be two copies of the algebras of $q$-difference operators with rational coefficients, i.e. operators of the type

$$
D_{q}^{N}+a_{1}(x) D_{q}^{N-1}+\cdots+a_{N}(x),
$$

where $D_{q}$ is the dilatation operator, $D_{q} f(x)=f(q x), q \in \mathbb{C}$, and $a_{i}(x)$ are rational functions. Let $\psi(x z)=\exp _{q}(x z)$ be the $q$-exponent (see e.g. [11)

$$
\exp _{q}(x)=\sum_{n=0}^{\infty} \frac{x^{n}}{(q ; q)_{n}},
$$

where $(q ; q)_{n}=(1-q)\left(1-q^{2}\right) \cdots\left(1-q^{n}\right),(q ; q)_{0}=1$. Let $\mathcal{B} \cong \mathcal{B}^{\prime}$ be two copies of the $q$-deformed Weyl algebra of operators

$$
\sum a_{j}(x) \partial_{q}^{j},
$$

where $\partial_{q}=\left(D_{q}-1\right) / x, a_{j}(x) \in \mathbb{C}[x]$. Put $b(x)=-\partial_{q}, b\left(\partial_{q}\right)=-x$. Then $\left(\mathcal{B}, \mathcal{B}^{\prime}, b\right)$ is a bispectral triple.

(ii) Let $L_{q, \beta}, \beta \in \mathbb{C}^{N}$, be the $q$-deformed Bessel operator:

$$
L_{\beta, q}=x^{-N}\left(D_{q}-q^{\beta_{1}}\right) \cdots\left(D_{q}-q^{\beta_{N}}\right) .
$$

One can find solution $\psi(x)$ to the equation

$$
L_{\beta, q} \psi=\psi
$$

of the form

$$
\psi(x)=x^{\beta_{k}} \sum_{j=0}^{\infty} a_{j} x^{j}, \quad a_{0}=1 .
$$

Define the algebra $\mathcal{B}$ to be generated by $D_{q}, x^{N}$ and $L_{\beta, q}$ and $\mathcal{B}^{\prime} \cong \mathcal{B}$ (in $\mathcal{B}^{\prime}$ the variable will be denoted by $z$ ). Then the anti-izomorphism will be

$$
\begin{aligned}
L_{\beta, q} & \mapsto z^{N} \\
b: \quad x^{N} & \mapsto L_{\beta, q} \\
D_{q} & \mapsto D_{q}
\end{aligned}
$$

with a joint eigenfunction $\psi(x, z)=\psi(x z)$.

Example 1.5 Let $\psi(x, n)=2^{-n} H_{n}(x), x \in \mathbb{C}, n \in \mathbb{Z}$, where $H_{n}$ are the Hermite polynomials (see e.g. [4]) for $n \geq 0$ and $H_{n}=0$ for $n<0$. Put $\mathcal{B}$ to be spanned by $x, \partial_{x}$ and $\mathcal{B}^{\prime}$ to be spanned by $n, T, T^{-1}$, where $T$ is the shift operator on functions defined on $\mathbb{Z}:(T f)(n)=f(n+1)$. Then an anti-isomorphism $b$ can be given by

$$
\begin{aligned}
b: \quad & x \mapsto\left(T+\frac{n}{2} T^{-1}\right) \\
& \partial \mapsto n T^{-1} .
\end{aligned}
$$

It is easy to check that the Hermite operator $\partial^{2}-2 x \partial$ is bispectral. One can repeat this construction for any set of orthogonal polynomials.

This example indicates that a general theory should work in various situations. In the next section we will see how to build new bispectral triples from a given one. 


\section{Automorphisms of algebras of differential operators}

In this section we will assume that the algebras $\mathcal{B}$ and $\mathcal{B}^{\prime}$ consist of differential operators. The main tool for constructing new bispectral operators will be the following simple observation.

Proposition 2.1 Let $\left(\mathcal{B}, \mathcal{B}^{\prime}, b\right)$ be a bispectral triple and let $L \in \mathcal{B}$. If $\operatorname{ad} L$ is a locally nilpotent operator $\operatorname{ad} L: \mathcal{B} \rightarrow \mathcal{B}$ then $\sigma=e^{\operatorname{ad} L}$ is an automorphism of $\mathcal{B}$ and $\left(\mathcal{B}, \mathcal{B}^{\prime}, b \circ \sigma\right)$ is a bispectral triple.

(Here locally nilpotent means that for each $M \in \mathcal{B}$ there exists $n$ such that $(\operatorname{ad} L)^{n} M=$ 0.) The proof of Proposition 2.1 being obvious is omitted.

An important example of an operator $L$ acting locally nilpotently on $\mathcal{B}$ is any bispectral operator [9]. This can be proved by using the bispectral anti-isomorphism $b$ and the fact that the function $f=b(L)$ acts locally nilpotently on $\mathcal{B}^{\prime}$.

Remark 2.2 (i) Obviously, exchanging the roles of $\mathcal{B}$ and $\mathcal{B}^{\prime}$ one can use also the anti-isomorphism $b_{2}=e^{\text {ad } L^{\prime}} \circ b$, where $L^{\prime}$ acts locally nilpotently on $\mathcal{B}^{\prime}$. We will use Proposition 2.1 in both versions.

(ii) In the definition of a bispectral triple a decisive role is played by the function $\psi(x, z) \in \mathcal{M}$ via which the anti-isomorphism is defined. Proposition 2.1 defines a new anti-isomorphism $b_{1}$ in terms of the old function $\psi$ (see (1.4)). But to have really a bispectral triple in the sense of Definition 1.1 we face the difficult task to define a new function $\tilde{\psi}$ to satisfy $P \tilde{\psi}=b_{1}(P) \tilde{\psi}$. The most natural choice $\tilde{\psi}=e^{L} \psi$ often makes no sense. In the examples below after defining the bispectral triple using $\psi$ the new function (or formal power series) $\tilde{\psi}$ can be determined using other arguments.

Although trivial the above Proposition is a source of new bispectral operators.

Example 2.3 (i) Let $\mathcal{B}$ and $\mathcal{B}^{\prime}$ be two copies of the Weyl algebra and let $b(x)=\partial_{z}$, $b\left(\partial_{x}\right)=z, \psi=e^{x z}$. For any polynomial $p(\xi)$ the operators $\operatorname{ad} p(x)$ and $\operatorname{ad} p\left(\partial_{x}\right)$ act locally nilpotently on $\mathcal{B}$. Then if we put $\sigma_{1}=e^{\operatorname{ad} p(x)}$ and $b_{1}=b \circ \sigma_{1}$ we have

$$
\begin{aligned}
& b_{1}(x)=b(x)=\partial_{z}, \\
& b_{1}\left(\partial_{x}\right)=b\left(-p^{\prime}(x)+\partial_{x}\right)=z-p^{\prime}\left(\partial_{z}\right) .
\end{aligned}
$$

In this way we get a new bispectral triple $\left(\mathcal{B}, \mathcal{B}^{\prime}, b_{1}\right)$ with a new function $\tilde{\psi}(x, z)=$ $e^{p^{\prime}(x)} e^{x z}$

(ii) One can apply the automorphism $\sigma_{2}=e^{\operatorname{ad} q\left(\partial_{x}\right)}$ to the triple $\left(\mathcal{B}, \mathcal{B}^{\prime}, b_{1}\right)$. Then we get $b_{2}=b_{1} \circ \sigma_{2}$, which gives

$$
\begin{aligned}
& b_{2}(x)=b_{1}\left(x+q^{\prime}\left(\partial_{x}\right)\right)=\partial_{z}+q^{\prime}\left(z-p^{\prime}\left(\partial_{z}\right),\right. \\
& b_{2}\left(\partial_{x}\right)=b_{1}\left(\partial_{x}\right)=z-p^{\prime}\left(\partial_{z}\right) .
\end{aligned}
$$

For the new function $\psi_{2}(x, z)$ we must have

$$
\begin{aligned}
& \partial_{x} \psi_{2}=\left(z-p^{\prime}\left(\partial_{z}\right)\right) \psi_{2}, \\
& x \psi_{2}=\left(\partial_{z}-q^{\prime}\left(z-p^{\prime}\left(\partial_{z}\right)\right)\right) \psi_{2} .
\end{aligned}
$$


This implies

$$
\left(\partial_{x}+p^{\prime}\left(x-q^{\prime}\left(\partial_{x}\right)\right)\right) \psi_{2}=z \psi_{2},
$$

which gives a new non-trivial example of bispectral operators, provided $\psi_{2}$ exists. One can construct a solution $\psi_{2}$ using Fourier transformation with respect to both $z$ and $x$ on $(2.5)$ and on

$$
x \psi_{2}=\left(\partial_{z}+q^{\prime}\left(\partial_{x}\right)\right) \psi_{2} .
$$

Because $\mathcal{K}_{\psi_{2}}=\mathbb{C}[x], \mathcal{K}_{\psi_{2}}^{\prime}=\mathbb{C}[z]$ we see that both spectral curves are isomorphic to $\mathbb{C}$.

Remark 2.4 The automorphisms considered in Example 2.3 generate the entire group of automorphisms of the Weyl algebra [7]. This result leads to a classification of all solutions to the bispectral problem such that $\mathcal{B}_{\psi} \cong \mathcal{B}_{\psi}^{\prime}$ is the Weyl algebra.

In general, we propose the following approach to the classification problem. Given solution $\psi$ to the bispectral problem is first reduced to a one with a "better" $\mathcal{B}_{\psi}$ (perhaps by a "bispectral Darboux transformation" - see the next section). In all known to us cases this $\mathcal{B}_{\psi}$ is either the Weyl algebra or $\left\langle x^{N}, D, L_{\beta}\right\rangle$ (see Example 1.3). Then the second step is for a given ring $\mathcal{B}$ to find all bispectral triples $\left(\mathcal{B}, \mathcal{B}^{\prime}, b\right)$.

One can easily check that the operators in the r.h.s. of (2.5) and (2.6) satisfy the so-called string equation $[P, Q]=1$, which plays an important role in $2 \mathrm{~d}$-quantum gravity [5, 8, 12] (see also 18, 15]). The corresponding to the r.h.s. of (2.5) taufunctions can be shown to satisfy the $W$-constraints (see [1, 10]). This will be done in another place.

An anti-isomorphism which can successfully be used for constructing new bispectral operators from old ones is the formal conjugation: $b(x)=x, b\left(\partial_{x}\right)=-\partial_{x}$. Instead of formulating a general result we shall give an example.

Example 2.5 Let $\beta \in \mathbb{C}^{N}, \gamma \in \mathbb{C}^{N}, \beta_{i}+\gamma_{i}=N-1$. Define $\mathcal{B}=\left\langle x^{N}, L_{\beta}, D_{x}\right\rangle$, $\mathcal{B}^{\prime}=\left\langle z^{N}, L_{\gamma}, D_{z}\right\rangle$, where the operators $L_{\beta}, L_{\gamma}$ are Bessel operators (see [3] or Example 1.3 (iii)). Let $b$ be the formal conjugation: $b\left(x^{N}\right)=z^{N}, b\left(D_{x}\right)=-D_{z}-1$, $b\left(L_{\beta}\right)=(-1)^{N} L_{\gamma}$. As $L_{\gamma}$ acts locally nilpotently on $\mathcal{B}^{\prime}$ (since it is bispectral) one can define $b_{1}=e^{-\operatorname{ad} N^{-1} L_{\gamma}} \circ b$. Then we have

$$
\begin{aligned}
& b_{1}\left(D_{x}\right)=-D_{z}-1+L_{\gamma}, \\
& b_{1}\left(L_{\beta}\right)=(-1)^{N} L_{\gamma}, \\
& b_{1}\left(x^{N}\right)=M_{\gamma}=e^{-\operatorname{ad} N^{-1} L_{\gamma}}\left(z^{N}\right) .
\end{aligned}
$$

$M_{\gamma}$ can be computed easily applying $b_{1}$ to both sides of the identity

$$
L_{\beta} x^{N}=\left(D_{x}+N-\beta_{1}\right) \cdots\left(D_{x}+N-\beta_{N}\right) .
$$

In the simplest nontrivial case $N=3$ we have

$$
M_{\gamma}=-L_{\gamma}^{2}+\left(3 D_{z}-9-\sum \gamma_{i}\right) L_{\gamma}-3 D_{z}^{2}+\left(9+2 \sum \gamma_{i}\right) D_{z}-\left(9+3 \sum \gamma_{i}+\sum \gamma_{i} \gamma_{j}\right)+z^{3} .
$$


The corresponding function $\psi(x, z)$ satisfies

$$
\begin{aligned}
& \left(D_{x}+D_{z}+1\right) \psi=L_{\gamma} \psi=(-1)^{N} L_{\beta} \psi, \\
& x^{N} \psi=M_{\gamma} \psi \\
& z^{N} \psi=M_{\beta} \psi
\end{aligned}
$$

For "generic" $\beta$ (see [3]) both spectral curves are $\mathbb{C}$.

This construction can be generalized considering $b_{1}=e^{\operatorname{ad} p\left(L_{\gamma}\right)} \circ b$ for arbitrary polynomial $p$.

Example 2.6 The last example deals with the non-generic $\beta=(-1,1,3)$. In this case the algebra $\mathcal{B}_{\psi}=\left\langle x^{2}, x^{3}, D_{x}, L_{\beta}\right\rangle$ is larger than for generic $\beta$ (cf. Example 1.3 (ii)). We have the anti-isomorphism

$$
\begin{aligned}
& x^{2} \mapsto z^{2}, \quad x^{3} \mapsto z^{3}, \\
b: \quad & D_{x} \mapsto-D_{z}-1, \\
& L_{\beta} \mapsto L_{\beta} .
\end{aligned}
$$

Let $b_{1}=e^{(-1 / 3) \operatorname{ad} L_{\beta}} \circ b$. We obtain the bispectral operator

$L=e^{(-1 / 3) \operatorname{ad} L_{\beta}}\left(x^{2}\right)=x^{-4}(D+1)(D-1)(D-2)(D-4)-2 x^{-1}(D+1)(D-1)+x^{2}$,

which can be written as

$$
L=\left(\partial^{2}-x^{-1} \partial-x\right)\left(\partial^{2}+x^{-1} \partial-x\right) .
$$

It coincides with a Darboux transformation of the Airy operator $\partial^{2}-x-$ see [3], Example 5.8 for $d_{0}=1, a=\infty, \lambda=\mu=0$ (cf. also [13], 14]).

The spectral algebra has rank 2 , the spectral curve is $\mathbb{C}$ with a cusp at 0 .

We hope the above examples help in unifying the Bessel and Airy cases of [3] which although similar still had important differences.

\section{Bispectral Darboux transformations}

Up to now the most widely used method for constructing solutions to the bispectral problem is the method of Darboux trasformations introduced in the pioneering work of Duistermaat and Grünbaum [9] (see also [19, 16, 2, 3, 14]). Here we give a general definition of bispectral Darboux transformations in the context of bispectal triples introduced in Sect. 1.

Definition 3.1 Let $\left(\mathcal{B}_{\psi}, \mathcal{B}_{\psi}^{\prime}, b\right)$ be a bispectral triple. Let $L \in \mathcal{A}_{\psi}$ be a bispectal operator which can be factorized as

$$
L=Q \theta^{-1} P \text { with } \quad P, Q \in \mathcal{B}_{\psi}, \theta \in \mathcal{K}_{\psi} .
$$

We call the operator

$$
\bar{L}=P Q \theta^{-1}
$$


a bispectral Darboux transformation of $L$ and the function

$$
\bar{\psi}=P \psi
$$

a bispectral Darboux transformation of $\psi$.

Theorem 3.2 The operator $\bar{L}$ is bispectral. More precisely, if $f=b(L) \in \mathcal{K}_{\psi}^{\prime}$ then

$$
\bar{L} \bar{\psi}=f \bar{\psi}
$$

and

$$
\bar{\Lambda} \bar{\psi}=\theta \bar{\psi}
$$

where

$$
\bar{\Lambda}=b(P) b(Q) f^{-1}
$$

Proof. Let $\Lambda=b(\theta)$. As $\mathcal{B}_{\psi}$ has no zero divisors (3.1) implies

$$
\theta=P L^{-1} Q, \quad L^{-1} \in \widetilde{\mathcal{A}}_{\psi} .
$$

Applying the anti-isomorphism $b$ we obtain

$$
\Lambda=b(\theta)=b(Q) f^{-1} b(P) .
$$

This shows that $\bar{\Lambda}$ is a bispectral Darboux transformation of $\Lambda$. From (3.2) and (3.6) it follows that $\bar{\psi}$ satisfies (3.4) and (3.5).

Theorem 3.2 is an abstract version of our bispectrality theorem from [3]. However, it formalizes only partially the arguments of this theorem. The main difference is that here we do not touch the subtle (in general) question when a factorization of the form (3.1) is possible. Nevertheless, Theorem 3.2 can be a part of a general theory of bispectral operators. Below we illustrate it by examples which have not appeared elsewhere.

Example 3.3 (i) Let $\left(\mathcal{B}, \mathcal{B}^{\prime}, b\right)$ be the bispectral triple from Example 1.4 (i), i.e. $\mathcal{B} \cong \mathcal{B}^{\prime}$ be two copies of the $q$-deformed Weyl algebra. Then $L=\partial_{q}^{2}$ is a bispectal operator with $\psi(x, z)=\exp _{q}(x z)$, i.e.

$$
\partial_{q}^{2} \exp _{q}(x z)=z^{2} \exp _{q}(x z)
$$

One can easily check that

$$
L=\left(\left(a+q^{2} x\right) \partial_{q}+q^{2}-1\right)((a+x)(a+q x))^{-1}\left((a+x) \partial_{q}-q+1\right),
$$

which gives the factorization of (3.1). Then if we put

$$
\psi_{1}(x, z)=\left((a+x) \partial_{q}-q+1\right) \exp _{q}(x z)
$$

Theorem 3.2 gives that

$$
\begin{aligned}
& \left((a+x) \partial_{q}-q+1\right)\left(\left(a+q^{2} x\right) \partial_{q}+q^{2}-1\right)((a+x)(a+q x))^{-1} \psi_{1}(x z)=z^{2} \psi_{1}(x z), \\
& \left(-z\left(a-\partial_{q}\right)-q+1\right)\left(-z\left(a-q^{2} \partial_{q}\right)+q^{2}-1\right) z^{-2} \psi_{1}(x z)=(a+x)(a+q x) \psi_{1}(x z) .
\end{aligned}
$$


(ii) Take the same bispectral triple but put

$$
L=\partial_{q}^{3}=x^{-3}(D-1)\left(q^{-1} D-1\right)\left(q^{-2} D-1\right) .
$$

For $\{a, b, c\}=\{0,1,2\} L$ can be factorized as $\left(q^{-a+3} D-1\right)\left(q^{-b+3} D-1\right) x^{-3}\left(q^{-c} D-\right.$ 1). The procedure of Theorem 3.2 gives the operator

$$
\bar{L}=x^{-3}\left(q^{-a} D-1\right)\left(q^{-b} D-1\right)\left(q^{-c-3} D-1\right) .
$$

It is clear that repeatedly applying this procedure one can get

$$
\widetilde{L}=x^{-3}\left(q^{-3 k} D-1\right)\left(q^{-3 m-1} D-1\right)\left(q^{-3 n-2} D-1\right)
$$

with any $k, m, n \geq 0$. Notice that $\widetilde{L}$ is a $q$-Bessel operator defined in Example 1.4 (ii).

(iii) Let $\left(\mathcal{B}, \mathcal{B}^{\prime}, b\right)$ be the bispectral triple defined in Example 1.4 (ii) and put

$$
L=L_{\beta, q}=x^{-2}\left(D-q^{\beta_{1}}\right)\left(D-q^{\beta_{2}}\right) .
$$

Let $\beta_{2}-\beta_{1}=2 \alpha, \alpha \in \mathbb{Z}_{\geq 0}$.

Then one can check that $L$ factorizes as $L=Q \theta^{-1} P$ with

$$
\begin{aligned}
Q & =q^{2} D\left(1+a q^{2 \alpha} x^{2 \alpha}\right)-q^{\beta_{2}}\left(1+a x^{2 \alpha}\right) \\
P & =\left(1+a x^{2 \alpha}\right) D-q^{\beta_{1}}\left(1+a q^{2 \alpha} x^{2 \alpha}\right) \\
\theta & =x^{2}\left(1+a x^{2 \alpha}\right)\left(1+a q^{2 \alpha} x^{2 \alpha}\right)
\end{aligned}
$$

for $a \in \mathbb{C}$. Applying the recipe of Theorem 3.2 one gets easily the bispectral operators $\bar{L}, \bar{\Lambda}:$

$$
\bar{L}=P Q \theta^{-1}, \quad \bar{\Lambda}=b(P) b(Q) f^{-1},
$$

satisfying (3.4, 3.5), where $f=z^{2}$ and

$$
\begin{aligned}
& b(P)=D\left(1+a L^{\alpha}\right)-q^{\beta_{1}}\left(1+a q^{2 \alpha} L^{\alpha}\right), \\
& b(Q)=\left(1+a q^{2 \alpha} L^{\alpha}\right) q^{2} D-q^{\beta_{2}}\left(1+a L^{\alpha}\right) .
\end{aligned}
$$

We end this section with the following observation. Suppose starting with a bispectral operator one produces a sequence of bispectral Darboux transformations labeled by some integer parameters $n_{1}, \ldots, n_{k}$. Suppose that all the operators $L_{n_{1}, \ldots, n_{k}}$ are of the same order and that their coefficients depend rationally on $n_{1}, \ldots, n_{k}$. Then considering $n_{1}, \ldots, n_{k}$ as arbitrary complex parameters we get a family of bispectral operators. The standard example is from [9] where from $\partial^{2}$ one produces $\partial^{2}-n(n-1) x^{-2}$ and putting $n(n-1)=c$ one gets arbitrary classical Bessel operator. Another example is provided by Example 3.3 (ii). The following is a non-trivial example illustrating this method.

Example 3.4 If $\psi(x, z)$ is the ordinary Airy solution of the bispectral problem, i.e.

$$
\begin{aligned}
& \left(\partial_{x}^{2}-x\right) \psi=z \psi, \\
& \partial_{x} \psi=\partial_{z} \psi, \\
& x \psi=\left(\partial_{z}^{2}-z\right) \psi,
\end{aligned}
$$


then $D_{z} \psi=\partial_{x}\left(\partial_{x}^{2}-x\right) \psi=\left(x^{-3} D_{x}\left(D_{x}-1\right)\left(D_{x}-2\right)-D_{x}-1\right) \psi$. From this it is easy to see that $\psi$ is the joint eigenfunction of Example 2.5 for $\beta=(0,1,2)$. For arbitrary $\beta \in \mathbb{C}^{3}$ denote that function by $\psi_{\beta}$. Then we have the following Darboux transformation:

$$
\psi_{\beta_{1}+1, \beta_{2}+1, \beta_{3}-2}=\frac{1}{z}\left(L_{\beta_{1}, \beta_{2}}\left(x, \partial_{x}\right)-x\right) \psi_{\beta_{1}, \beta_{2}, \beta_{3}} .
$$

For example the identity

$$
D_{z} \psi_{\beta_{1}+1, \beta_{2}+1, \beta_{3}-2}=\left(L_{\beta_{1}+1, \beta_{2}+1, \beta_{3}-2}\left(x, \partial_{x}\right)-D_{x}-1\right) \psi_{\beta_{1}+1, \beta_{2}+1, \beta_{3}-2}
$$

is a straightforward consequence of the equation

$$
\left(L_{\beta_{1}, \beta_{2}}-x\right)\left(L_{\beta_{1}, \beta_{2}, \beta_{3}}-D-2\right)=\left(L_{\beta_{1}+1, \beta_{2}+1, \beta_{3}-2}-D-1\right)\left(L_{\beta_{1}, \beta_{2}}-x\right) .
$$

In this way we obtain the bispectrality of Example 2.5 for $\beta=(i, 1+j, 2-i-j)$, where $i, j$ are arbitrary integers. Because of the polynomial dependence on $\beta$ of the above differential operators we can continue the joint eigenfunction for arbitrary $\beta \in \mathbb{C}^{3}$ such that $\beta_{1}+\beta_{2}+\beta_{3}=3$. Note that in Example 2.5 one can normalize $\beta_{1}+\beta_{2}+\beta_{3}$ by multiplying $\psi_{\beta}$ by $x^{c} z^{-c}$ for $c \in \mathbb{C}$ (cf. [3]).

The last Example together with Examles 2.5 and 2.6 shows that there is a connection among all methods presented here.

\section{Acknowledgement}

We thank Alex Kasman for stimulating correspondence and for sending us his preprint [14]. This work was partially supported by Grant MM-523/95 of Bulgarian Ministry of Education, Science and Technologies.

\section{References}

[1] M. Adler, P. van Moerbeke, Commun. Math. Phys. 147 (1992) 25.

[2] B. Bakalov, E. Horozov, M. Yakimov, Bäcklund-Darboux transformations in Sato's Grassmannian. Sofia preprint (1996), q-alg/9602010.

[3] B. Bakalov, E. Horozov, M. Yakimov, Bispectral algebras of commuting ordinary differential operators. Sofia preprint (1996), q-alg/9602011.

[4] H. Bateman, A. Erdélyi, Higher transcendental functions. New York: McGrawHill, 1953.

[5] E. Brézin, V. Kazakov, Phys. Lett. B 236 (1990) 144.

[6] J. L. Burchnall, T. W. Chaundy, Proc. Lond. Math. Soc. 21 (1923) 420; Proc. Royal Soc. London (A) 118 (1928) 557; Proc. Royal Soc. London (A) 134 (1932) 471.

[7] J. Dixmier, Bull. Soc. Math. France 96 (1968) 209.

[8] M. Douglas, S. Shenker, Nucl. Phys. B 335 (1990) 635.

[9] J. J. Duistermaat, F. A. Grünbaum, Commun. Math. Phys. 103 (1986) 177. 
[10] M. Fukuma, H. Kawai, R. Nakayama, Commun. Math. Phys. 143 (1992) 371.

[11] G. Gasper, M. Rahman, Basic hypergeometric series. Encyclopedia of Math. and its Appl. 35, Cambridge Univ. Press, 1990.

[12] D. J. Gross, A. Migdal, Phys. Rev. Lett. 64 (1990) 127.

[13] F. A. Grünbaum, Comm. Pure Appl. Math. 47 (1994) 307.

[14] A. Kasman, M. Rothstein, Bispectral Darboux transformations: The generalized Airy case. Univ. of Georgia preprint (1996).

[15] M. Kontsevich, Commun. Math. Phys. 147 (1992) 1.

[16] F. Magri, J. Zubelli, Commun. Math. Phys. 141 (1991) 329.

[17] G. Wilson, J. Reine Angew. Math. 442 (1993) 177.

[18] E. Witten, Surveys in Diff. Geom. 1 (1991) 243.

[19] J. Zubelli, Physica D 43 (1990) 269. 